\title{
Atenção ao parto e nascimento em maternidades do Norte e Nordeste brasileiros: percepção de avaliadores da Rede Cegonha
}

\author{
Labor and childbirth care in maternity facilities in Brazil's North \\ and Northeast regions: perceptions of the evaluators of the Stork \\ Network Program
}

\author{
Zeni Carvalho Lamy (https://orcid.org/0000-0002-9332-0542) ${ }^{1}$ \\ Laura Lamas Martins Gonçalves (https://orcid.org/0000-0003-2213-095X) ${ }^{1}$ \\ Ruth Helena de Souza Britto Ferreira de Carvalho (https://orcid.org/0000-0003-1180-1586) ${ }^{1}$ \\ Maria Teresa Seabra Soares de Britto e Alves (http://orcid.org./0000-0002-4806-7752) ${ }^{1}$ \\ Maria Eduarda Koser (http://orcid.org./0000-0001-7685-8669) ${ }^{1}$ \\ Matheus de Sousa Martins (http://orcid.org./0000-0001-7061-1665) ${ }^{1}$ \\ Neide Pires Leal (http://orcid.org./0000-0001-8793-1112) ${ }^{2}$ \\ Erika Barbara Abreu Fonseca Thomaz (http://orcid.org./0000-0003-4156-4067) ${ }^{1}$
}

${ }^{1}$ Programa de PósGraduação em Saúde Coletiva, Departamento de Saúde Pública, Universidade Federal do Maranhão. R. Barão de Itapary 155, Centro. 65020-070 São Luís MA Brasil. zenilamy@gmail.com ${ }^{2}$ Escola Nacional de Saúde Pública, Fiocruz. Rio de Janeiro RJ Brasil.

\begin{abstract}
This article eevaluates delivery and birth care practices in maternity facilities in Brazil's North and Northeast regions. We conducted a qualitative evaluation of 91 facilities in the North and 181 facilities in the Northeast. The data was collected using systematic observation by a team of 44 previously trained evaluators and recorded in a field diary. A thematic analysis of the collected data was performed, resulting in three core themes: challenges of collegial management; challenges for coping with obstetric violence; and the potential of the evaluation process for driving change. Advances were made in the implementation of good labor and childbirth care practices; however, some maternity facilities still reproduce hierarchical models without spaces for collegial management and accounts of obstetric violence were common. Health professionals used the presence of risk to justify the low level of adoption of good practices. However, the findings reveal progress towards the humanization of care. The results also show the potential of the evaluation process for driving change. Although progress has been made towards the adoption of the good practices recommended by the Stork Network Program both in the area of management and care delivery, many challenges remain in view of the dominance of a hierarchical management model associated with an interventionist approach to health care.
\end{abstract}

Key words Health evaluation, Humanized birth, Violence
Resumo O objetivo deste artigo é avaliar práticas de atenção ao parto e nascimento em maternidades do Norte e Nordeste brasileiros. Avaliação qualitativa realizada em 91 maternidades no Norte e 181 no Nordeste do Brasil. A técnica de pesquisa foi a observação sistematizada, realizada por 44 avaliadores previamente treinados e registrada em diário de campo. Foi realizada análise de conteúdo na modalidade temática. Os núcleos temáticos encontrados foram: Desafios da Gestão Colegiada; Desafios para o enfrentamento da Violência Obstétrica; e Potencial do processo avaliativo na indução de mudanças. Foram identificados avanços na implantação de boas práticas na gestão do cuidado e atenção à saúde, embora algumas maternidades ainda reproduzam um modelo hierárquico, sem espaços colegiados de gestão e com práticas de violência obstétrica. Situações de risco em gestantes justificaram menor adesão dos profissionais de saúde às boas práticas, ainda que ações em direção à humanização também tenham sido visibilizadas. Identificou-se a potência do processo avaliativo na indução de mudanças. Foram evidenciadas mudanças em direção às boas práticas preconizadas pela Rede Cegonha, tanto na gestão quanto na atenção, mas são muitos os desafios frente ao predominio de um modelo de gestão hierárquico associado a um modelo de atenção com práticas intervencionistas.

Palavras-chave Avaliação em saúde, Parto humanizado, Violência 


\section{Introdução}

A assistência humanizada ao parto é um dos pilares da Rede Cegonha (RC), instituída, em 2011, como política pública pelo Ministério da Saúde (MS), com o objetivo de reduzir a mortalidade materna e neonatal a partir da promoção de um modelo qualificado e humanizado de atenção ${ }^{1,2}$.

A RC se contrapõe à lógica do modelo hegemônico, caracterizado por alta frequência de cesariana, medicalização do parto e nascimento, abortos inseguros e taxas elevadas de morbimortalidade perinatal. É voltada para a promoção de partos e nascimentos saudáveis, garantindo privacidade, autonomia e protagonismo da mulher, sem intervenções desnecessárias ${ }^{3}$. Tais mudanças envolvem aspectos ligados ao processo de trabalho, a relações mais igualitárias entre gestão e assistência, entre categorias profissionais e entre profissionais e usuárias ${ }^{4}$.

Para a implementação desse modelo, todavia, há entraves relacionados ao modo de organização hospitalar, centrado no saber-poder biomédico, com hierarquia entre as categorias profissionais da saúde e sem o reconhecimento da autonomia e da subjetividade da mulher ${ }^{5}$.

Um dos desafios da RC foi vincular às estratégias de mudanças de práticas assistenciais, um processo sistematizado de monitoramento e avaliação das maternidades em todo o país com base nos objetivos, diretrizes, componentes e fases das Portarias e documentos que regulamentam a $\mathrm{RC}^{1}$. Nesse sentido, entre 2013 e 2015 foi realizado o $1^{\text {o }}$ ciclo avaliativo das maternidades contempladas nos Planos de Ação Regionais (PAR) ${ }^{6}$ e, entre 2016 e 2017, o $2^{\circ}$ ciclo avaliativo ${ }^{7,8}$. Os dados ora analisados dizem respeito a este último período.

Considerando as maiores desigualdades de cobertura e acesso à atenção em saúde nas regiões Norte (N) e Nordeste (NE) e, em consequência, a histórica persistência de altos índices de mortalidade materna e infantil, especialmente quando comparadas ao Sul e Sudeste, destaca-se um investimento mais efetivo da RC nessas regiões, expresso, inclusive, pela realização desta pesquisa. Assim, no período de 2010-2015, portanto, antes e após a implantação da RC, houve, no Brasil, uma redução de $11,6 \%$ na razão de mortalidade materna e de $15,6 \%$ na taxa de mortalidade infantil9.

Este artigo teve por objetivo avaliar as práticas de atenção ao parto e nascimento em maternidades do N e NE brasileiros na percepção de avaliadores da RC, buscando melhor compreensão dos desafios presentes nos serviços.

\section{Método}

Trata-se de uma avaliação qualitativa de serviços de saúde, vinculada ao projeto "Pesquisa avaliativa da gestão e atenção ao parto e nascimento na Rede SUS do Brasil" 7,8 .

Este estudo avaliou estabelecimentos de saúde públicos e privados conveniados ao SUS, das regiões $\mathrm{N}$ e NE que realizaram 500 ou mais partos em Região de Saúde com PAR da RC, independente de repasse direto de incentivo $(\mathrm{n}=$ 245); estabelecimentos que realizaram menos de 500 partos, com incentivos da RC vinculados ao custeio de leitos $(n=15)$; e aqueles que não faziam parto, mas receberam recursos para leitos de Unidades Neonatais $(n=12)$. Para identificar os estabelecimentos elegíveis utilizamos os dados do Sistema de Informações Hospitalares do SUS, referentes ao ano de 2015, que, à época, eram os mais atuais consolidados e disponíveis para consulta.

No total, foram avaliadas 272 instituições de saúde, sendo 181 no NE: Maranhão (14), Pernambuco (32), Piauí (8), Ceará (44), Rio Grande do Norte (14), Paraíba (10), Alagoas (16), Sergipe (9), Bahia (34); e 91 no N: Acre (6), Amapá (4), Amazonas (12), Pará (54), Rondônia (7), Roraima (1) e Tocantins (7).

Para a realização do trabalho de campo foram selecionados 44 profissionais ( 28 avaliadores para o NE e 16 para o N) com atuação em saúde materna e infantil (gestão e/ou assistência) para participação em capacitação de 70 horas, com duração de duas semanas. A pesquisa também contou com 10 supervisores que atuavam no suporte aos avaliadores e na articulação com gestores das maternidades e das secretarias estaduais e municipais.

Os dados foram coletados de dezembro/2016 a junho/2017. No primeiro dia de avaliação, em cada serviço, era realizada uma reunião com os gestores locais e regionais (diretores e chefes de serviço de obstetrícia e neonatologia, secretários de saúde e representantes das regionais de saúde dos estados), com a finalidade de explicar os objetivos e a metodologia da avaliação. Os avaliadores trabalharam em duplas e permaneciam, em cada serviço, pelo período de três dias, nos estabelecimentos do NE, e de cinco dias nos do N.

A técnica de coleta de dados foi a observação sistemática, realizada durante a permanência no campo, a partir de um roteiro previamente elaborado que buscou captar as diferentes perspectivas dos sujeitos investigados, incluídas suas ambiguidades e tensões. 
O roteiro continha questões relativas a: 1) informações gerais da maternidade; 2) uso de protocolos e indicadores; 3 ) atenção e cuidado com mulheres e recém-nascidos; 4) qualidade da informação e acolhimento ofertados às mulheres e acompanhantes; 5) postura dos gestores em relação ao modelo institucional de atenção ao parto e nascimento e adesão às boas práticas; 6) participação dos trabalhadores nos espaços de decisão, institucionalizados ou informais; e 7) comunicação entre gestores e trabalhadores quanto à organização, processo e condições de trabalho. As informações obtidas na observação foram registradas em diário de campo.

Foi realizada análise de conteúdo na modalidade temática ${ }^{10}$. A compreensão da realidade em análise se deu a partir dos referenciais teóricos de processo de trabalho, humanização da assistência e implantação de boas práticas obstétricas e neonatais. Foram consideradas relações de poder estabelecidas, formas de organização do processo de trabalho e condições de trabalho.

A pesquisa foi aprovada pelo Comitê de Ética em Pesquisa com seres humanos da Universidade Federal do Maranhão e da Escola Nacional de Saúde Pública Sérgio Arouca. Para garantir o sigilo, a identificação dos estabelecimentos de saúde foi substituída por código contendo $\mathrm{N}$ ou NE, um número para cada estado, seguido de outro para cada estabelecimento.

\section{Resultados e discussão}

A partir da análise dos diários de campo, foram apreendidos os seguintes núcleos temáticos: 1) Desafios da gestão colegiada; 2) Desafios para o enfrentamento da violência obstétrica; e 3 ) Potencial do processo avaliativo na indução de mudanças.

\section{Desafios da gestão colegiada}

Foram encontrados estabelecimentos com o dispositivo de gestão colegiada implantado ou em implantação e com espaços decisórios efetivamente compartilhados, indicando importante avanço em direção a mudanças do modelo de gestão, como preconizado pela RC.

A instituição tem espaços de gestão compartilhada, onde são discutidos casos e rotinas de trabalho, com o intuito de aprimorar a assistência prestada às usuárias. A partir das discussões de casos, são avaliadas as condutas. Regularmente os funcionários participam de cursos de capacitação e atualização pertinentes à área de atuação. (N3-6)
Não existe um colegiado, mas está em processo de organização. Porém, há rodas de conversas feitas mensalmente onde todos os profissionais são convidados e, em geral, participam. Existem capacitações e os próprios profissionais buscam se capacitar, pois são estimulados e empenhados na adequação do processo de trabalho. (N6-7)

Fragilidades nos processos de gestão colegiada já foram apontadas desde o primeiro ciclo avaliativo da RC. Os espaços coletivos de interlocução como Grupos Condutores, Colegiados Gestores e Fóruns foram reconhecidos como mecanismos de fortalecimento de espaços compartilhados de decisão, porém, ainda pouco implantados. Além disso, as situações de mudança e descontinuidade nas gestões estaduais, municipais e hospitalares também foram apontadas como motivos para retrocessos nos processos de implementação da $\mathrm{RC}^{6}$.

Houve casos, ainda, de serviços sem espaços colegiados de gestão e outros em que, mesmo apresentando colegiados implantados, foi identificada uma reprodução das mesmas práticas centralizadoras e excludentes da participação de profissionais e usuárias. Relatos dos diários de campo demonstram as diversas situações encontradas:

Existem reuniões entre a direção e os coordenadores das unidades para discussões do processo de trabalho, porém não incluem os profissionais da assistência de forma sistematizada. Somente de forma pontual e informal. (NE2-20)

As decisões colegiadas não envolvem os técnicos, tampouco as usuárias. Parece que funcionam por temática, como, por exemplo, a de mortalidade materna; e as demais reuniões são feitas por categorias profissionais separadas. (NE2-23)

Apesar do avanço em serviços com instâncias de gestão colegiada, de modo geral, permanece a clássica divisão do trabalho entre os que planejam e os que executam as ações, separando gestão e atenção. Nesta forma de trabalho, cabe à primeira a tomada de decisão acerca do planejamento e da organização do processo de trabalho ${ }^{11,12}$, e, à segunda, o exercício das práticas cotidianas de atenção. Os trabalhadores, principais envolvidos na atenção, não participavam dos processos decisórios. Além disso, cada categoria profissional cuidava do seu núcleo específico, reproduzindo essa fragmentação e estabelecendo uma hierarquia de poder.

O debate sobre os modos de gestão do trabalho em saúde não é novo e evidencia a necessidade de interrogar uma racionalidade administrativa hegemônica nos serviços que restringe 
espaços de inventividade e fortalece mecanismos de controle e assujeitamento tanto de profissionais quanto de usuários ${ }^{13}$.

A diretriz da Gestão Participativa e Compartilhada afirma a inseparabilidade entre os processos de gestão e atenção, pois a gestão dos processos de trabalho em saúde não pode ser compreendida como tarefa administrativa separada das práticas de cuidado ${ }^{14}$. O modo como é organizado o processo de trabalho interfere diretamente na atenção prestada. Manter a subordinação de trabalhadores aos gestores nos espaços colegiados implica restringir a dinâmica de comunicação à mera transmissão de decisões, atualizando as relações de poder estabelecidas entre as diversas categorias profissionais constituintes do campo da saúde.

O Colegiado Gestor é um dispositivo de humanização que considera a gestão do processo de trabalho um desafio coletivo $^{13}$. Esse dispositivo tem potencial para produzir mudanças, avançando no desafio de democratização dos serviços, a partir da inclusão de novos sujeitos para produção de corresponsabilidade nos modos de gerir e cuidar $^{15}$.

A implementação dos dispositivos de humanização, por si só, não garante a participação dos diferentes agentes implicados no processo de produção de saúde ${ }^{16}$. Isso significa que o grupo integrante do Colegiado Gestor precisa avaliar seu próprio modo de funcionamento e os processos de trabalho no cotidiano dos serviços.

Para melhorar a comunicação entre trabalhadores e gestores, os avaliadores, em suas narrativas, apontaram o uso das novas tecnologias de comunicação no cotidiano de alguns serviços como ferramenta de trabalho. O e-mail e o WhatsApp ${ }^{\circledR}$ foram usados como canais de comunicação.

Em geral, percebemos uma boa relação entre gestores e trabalhadores. Os profissionais fazem menções muito positivas a respeito da gestão, que visivelmente é bastante ativa. A comunicação ocorre abertamente via grupos de WhatsApp ${ }^{\circledR}$, de e-mails, reuniões, capacitações etc. Embora não existam espaços formalizados de gestão compartilhada, os profissionais, de modo geral, sentem que suas questões são levadas em consideração na tomada de decisão da gestão. (NE2-26)

Embora, em algumas situações, o WhatsA$\mathrm{pp}^{\circledR}$ fosse usado para facilitar o compartilhamento das decisões, em outras, era evidente, para os avaliadores, a reprodução da dinâmica institucional, servindo como mero canal de difusão das decisões tomadas pelos gestores e não como espaço para decisões coletivas.
Existem reuniões entre os coordenadores das unidades e algumas informações são passadas para os profissionais em grupos de WhatsApp ${ }^{\circledR}$ (principalmente para os médicos), mas eles não participam e não são informados das discussões realizadas nas reuniões de gestão. (NE4-3)

A ampliação do grau de comunicação intra e intergrupos vem sendo apontada como de fundamental importância no exercício de uma maior democracia institucional nos serviços. Favorece a capacidade de interferência mútua entre os sujeitos e sua capacidade de produzir novos sentidos para e no trabalho. Uma organização do trabalho mais coletiva reflete diálogo e troca entre os profissionais, favorecendo a qualidade da atenção e a definição mais clara de corresponsabilidades entre os profissionais e equipes ${ }^{5}$.

Uma justificativa apresentada pelos gestores para a pequena participação dos trabalhadores nas reuniões é a condição de plantonista. Essa é uma questão crucial a ser considerada no enfrentamento da estrutura e da lógica de organização dos hospitais, pois, para que haja compartilhamento de decisões e corresponsabilidade no cuidado, os trabalhadores precisam se encontrar e construir um cotidiano de trocas. Não há mudança nos modelos de gestão e atenção sem mudança nos processos de trabalho e, para isso, é necessário também repensar a organização. A implementação da horizontalização do cuidado é um tema que precisa ser enfrentado com a constituição de equipes de referência ${ }^{17}$.

Igualmente importante é o tema da rotatividade de trabalhadores, que aparece nas narrativas dos avaliadores. A valorização do trabalho e do trabalhador da saúde, a partir de vínculos trabalhistas e condições não precarizadas de trabalho, é importante para a promoção da saúde do trabalhador, para a fixação dos profissionais e para a qualificação do cuidado ${ }^{18}$.

\section{Desafios para o enfrentamento da violência obstétrica}

Um dos grandes investimentos da RC tem sido propor estratégias para a redução de situações de violência obstétrica. Problematizar práticas e atitudes naturalizadas no cotidiano dos serviços, contrárias à perspectiva da humanização da assistência, tem sido um importante passo na direção de mudanças na atenção à saúde materna e infantil.

Imprecisões conceituais sobre a violência obstétrica, inclusive em termos legais, inviabilizam a criminalização desse tipo de violência, a despeito 
de evidências de sua prática ${ }^{19}$. No plano internacional, o termo violência obstétrica está associado à violação de direitos humanos das mulheres, sendo reconhecido como questão de saúde pública pela Organização Mundial da Saúde (OMS) ${ }^{20}$. Os maus-tratos e as violências sofridos no parto configuram-se como práticas generalizadas, em sistemas de saúde de diversos países, e dizem respeito a condições de risco, abusos e descasos ${ }^{21,22}$. No âmbito nacional, tem sido entendida como uma dimensão da violência institucional ${ }^{23}$, mas, também, de gênero ${ }^{24}$, sendo a RC uma das estratégia para o enfrentamento dessa realidade.

Esse processo que vai desde o reconhecimento da violência até a efetivação de mudanças de práticas arraigadas no cotidiano dos serviços é um percurso lento, com avanços e recuos. Transformações em direção às boas práticas coexistem com situações de violência identificadas e descritas pelos avaliadores, tanto em estabelecimentos do N como do NE.

Há relatos de violência obstétrica pelas puérperas e se percebe um medo em suas falas. Uma vez que todos se conhecem e o hospital é o único do município [...], [elas] têm medo de represálias. (N4-9)

Algumas puérperas se queixaram do atendimento. Foram maltratadas por técnicas de enfermagem que reclamavam dos gritos das parturientes e mandavam elas "calarem a boca" e que deviam ficar quietas no leito. (N4-11)

Quando há laceração ou episiotomia, as mulheres precisam se deslocar andando para a sala de procedimento que fica no final do corredor dos boxes improvisados para rafia. (N5-2)

Dentre as situações descritas, o diagnóstico de "risco obstétrico" justificou menor adesão às boas práticas por parte de profissionais de hospitais de referência para o atendimento de gestantes de alto risco. Para gestores e trabalhadores, um parto de alto risco torna-se um ato médico.

Existe uma crença de que o parto de alto risco não se relaciona com o modelo de humanização $e$ todas as intervenções são justificadas por se tratar de uma maternidade de alto risco. (NE2-15)

Na perspectiva de profissionais de saúde, o binômio risco-segurança autoriza intervenções sem explicações ou solicitações, pois entendem que a realização de procedimentos sem consentimento não caracteriza a violência, mas, sim, a garantia de segurança no parto ${ }^{25}$. Este tipo de cuidado em saúde privilegia a hegemonia médica e o cuidado técnico em detrimento do direito das usuárias.

A atribuição de risco à mulher no ciclo gravídico-puerperal tem a finalidade de controlar efeitos de morbimortalidade futura e sua utili- zação estabelece diferenças entre as gestações. Para Robles ${ }^{26}$, o risco é uma categoria reguladora na saúde materna e infantil utilizada para legitimar o uso de tecnologias duras e de intervenções médicas vistas como forma de controle sobre as doenças.

A institucionalização do abuso e desrespeito à autonomia da mulher é naturalizada diante da necessidade de salvar vidas. Pesquisas têm apontado a existência de maus-tratos à mulher em estabelecimentos que atendem a partos e nascimentos em diferentes contextos ${ }^{27-29}$. O uso excessivo de medicalização no processo natural de parir, tem sido apontado como uma prática cotidiana, caracterizando violência obstétrica, desrespeito/abuso, impetrado pelos profissionais de saúde ${ }^{28}$. A ideia de que o parto é arriscado em si mesmo, e de que não há gravidez sem riscos, como o termo "risco habitual" indica, pode estar na gênese desses abusos e explicar os resultados identificados nesta avaliação.

No contexto da formação médica no Brasil e na América Latina, a violência contra a mulher está presente, como ferramenta pedagógica, no ensino de procedimentos dolorosos e desnecessários, mas naturalizados, a partir de estereótipos de gênero, e incorporados aos processos de trabalho ${ }^{30}$. Segundo Diniz et al. ${ }^{31}$, é ensinado aos profissionais que as parturientes não têm direito de escolha e que a necessidade de ensino é mais importante que a autonomia ou a integridade das parturientes, banalizando a violação dos direitos das mulheres, constituindo-se como parte oculta do currículo.

Relatos dos avaliadores indicaram que em hospitais de ensino, inclusive universitários, a garantia de aulas práticas para os alunos, muitas vezes, foi colocada à frente das necessidades de saúde e dos direitos das mulheres.

Muitas mulheres relataram incômodo com residentes e estudantes. Os locais onde a assistência é prestada está sempre repleta de estudantes. Alguns se comportam de maneira inadequada, causando sofrimento para as mulheres. (NE2-14)

A maternidade recebe instituições de ensino para realização de estágio supervisionado. No tocante à medicina, mulheres recebem intervenções desnecessárias para que o residente/estagiário aprenda procedimentos, causando aumento do número de episiotomias e também cesáreas desnecessárias, além de repetitivos exames de toque. (NE8-8)

Estudantes fazem toques sequenciais sem pedir permissão. As mulheres se sentem na obrigação de servirem de objeto de estudo. Uma mulher falou: 
'porque é o trabalho deles e eu não posso reclamar'. (NE2-27)

O hospital recebe estudantes $e$, por vezes, um dos médicos dá aulas para os estudantes da graduação dentro da UCINCO. (NE 4-10)

Os relatos acima, que apontam violência obstétrica, têm sido discutidos em outras pesquisas, sem que esse termo seja explicitado. Uma pesquisa acerca da violência institucional praticada por profissionais de saúde em maternidades aponta que, embora muitos reconheçam o tratamento grosseiro e desrespeitoso com as mulheres, tais atitudes são banalizadas por profissionais que as encaram, inclusive, como "brincadeiras". O argumento para justificar a adoção de condutas violentas, como expressões pejorativas, ameaças e reprimendas às parturientes, é a necessidade do uso de autoridade para manejar situações corriqueiras do serviço ${ }^{28}$. Chama-se a atenção da importância do termo violência obstétrica quando a violência institucional for praticada na assistência à mulher durante o parto e nascimento.

A falta de estrutura e de recursos humanos, assim como a superlotação, foram outras questões apontadas pelos profissionais como responsáveis pela violação de direitos:

Existe apenas um banheiro no pré-parto que estava muito sujo e com fila de mulheres em trabatho de parto para utilizá-lo. As salas de parto normal ficam dentro do Bloco Cirúrgico e as mulheres após o parto ficam em macas nos dois lados do corredor sem conseguir leito no alojamento conjunto. (NE2-27)

Não podemos falar que existem boas práticas, a maternidade está rotineiramente superlotada. As mulheres não têm nenhuma privacidade e não podem entrar com acompanhante, nem no pré-parto, nem na sala de parto, nem no pós-parto. (NE2-27)

Falta também equipe suficiente para prestar uma assistência de qualidade. O subdimensionamento das equipes impede que várias boas práticas sejam adotadas, porque os profissionais têm pouco tempo para atender cada usuária. (NE4-5)

Há dificuldades de incorporação das boas práticas pelos profissionais dos diferentes setores, principalmente pela superlotação e ritmo acelerado dos trabalhos. A relação dos profissionais com as puérperas também é permeada por descaso e violência. (NE2-23)

A maternidade não permite a presença de acompanhante durante todo o processo do trabatho de parto, justificada pela superlotação e falta de privacidade. (NE9-8)

Essas questões levam à consequente sobrecarga, em intensidade e ritmo, e contribuem para a precarização das condições de trabalho. $\mathrm{O}$ atendimento a uma demanda acima de sua capacidade operacional, equipes profissionais subdimensionadas e sem acesso à educação permanente, constituem elementos na conformação das condições de trabalho a que estão submetidos os profissionais das instituições analisadas.

A superlotação produziu, também, desentendimentos entre a equipe, entre equipe e usuárias e entre as próprias usuárias e familiares. Esta situação teve efeitos na qualidade da atenção prestada às usuárias e familiares, dificultando direito a acompanhante, oferta de métodos não farmacológicos para a dor e, até mesmo, informações sobre sua situação de saúde e do filho. Desde 2014, essas práticas foram incluídas como Cuidado Amigo da Mulher na habilitação de hospitais na Iniciativa Hospital Amigo da Criança, dentre outras ${ }^{32}$.

Elas não recebem informações sobre direito ao acompanhante, sobre ouvidoria, sobre qualquer direito que seja. E são poucas as informações sobre a situação de saúde delas. Muitas mulheres relataram: 'minha mãe, meu marido ou minha acompanhante só souberam que meu bebê tinha nascido várias horas depois'. (NE2-27)

As puérperas tinham poucas informações sobre suas condições do parto e dos cuidados ao recémnascido. (NE9-20)

São muitas as situações de violação dos direitos das mulheres, a partir de práticas que indicam relações de poder que as despersonalizam, reduzindo-as à condição de objeto de intervenção ${ }^{25,27,31}$.

$\mathrm{Na}$ contramão de tais práticas, avaliadores indicaram também a presença de instituições em que gestores e profissionais encontravamse engajados no processo de implementação de mudanças a favor da não violência e em defesa dos direitos das usuárias. Em tal processo de mudança coexistem práticas mais ou menos próximas da práxis da humanização, expressando as tensões presentes nas relações entre gestores e profissionais e entre estes e usuárias e familiares.

Foi perceptivel, durante o processo de avaliação da maternidade, ações que indicam o compromisso da gestão, bem como dos trabalhadores, com as boas práticas na atenção ao parto e nascimento, convergindo, portanto, com a proposta da Rede Cegonha. (NE2-13)

Presença de acompanhante em tempo integral. Oferta de métodos não farmacológicos para manejo da dor. Livre deambulação. Oferta de líquidos $e$ alimentos durante o trabalho de parto. Contato pele-a-pele logo após o nascimento é rotina bem 
estabelecida na Maternidade. [...] Não há acolhimento com classificação de risco implantado. Após a admissão, as mulheres são examinadas na sala de pré-parto sem a devida privacidade. A gestão parece pouco integrada com os trabalhadores. (NE3-8)

O desenvolvimento das boas práticas ao parto e nascimento já é uma rotina, estranham quando a mulher não deseja, mas sempre respeitam a escolha da mulher. Possuem doulas voluntárias que também apoiam a realização das boas práticas, empoderando as mulheres no momento do parto. (NE9-14)

A gestão está bem empenhada com as mudanças de modelo de atenção ao parto e nascimento. (N1-4)

Essa realidade revela a complexidade do processo de mudanças voltadas às boas práticas $e$ necessidade de continuação do processo das mudanças produzido pela RC. Apesar dos avanços alcançados, investimentos na formação e educação continuada para trabalhadores de saúde ainda são necessários, assim como maior divulgação de informações para as mulheres, de modo a conhecerem seus direitos.

\section{Potencial do processo avaliativo na indução de mudanças}

A partir dos diários de campo dos avaliadores foi possível identificar a potência da metodologia avaliativa da RC na indução de mudanças nos serviços. Neste segundo ciclo avaliativo, foram identificados avanços desencadeados pelo primeiro ciclo de avaliações da RC.

Foi relatada, na entrevista com gestores, a avaliação anterior [ocorrida em 2015, no primeiro ciclo], em que a maternidade ficou com vários pontos baixos. A partir de então, procuraram melhorar seus indicadores. (NE2-13)

Dessa forma, foi apontado o potencial transformador do monitoramento da RC. No primeiro ciclo, os avaliadores relataram que o preenchimento do instrumento avaliativo suscitava discussões sobre as potencialidades e as fragilidades do serviço, com proposição de ações para a melhoraria dos indicadores ${ }^{6}$. Um dos objetivos da avaliação da RC é justamente induzir o desenvolvimento de um conjunto de ações que permitam mudanças no modelo de gestão e atenção à saúde nas maternidades do SUS ${ }^{33}$.

No segundo ciclo, os gestores eram informados previamente da lista de protocolos e indicadores que seriam solicitados. Saber que seriam avaliados levava a uma preparação que, em muitas situações, induzia mudanças.
Em muitos casos, apesar da entrega dos protocolos, ficava evidente aos avaliadores que não eram de uso cotidiano e que tinham sido impressos apenas para serem entregues à equipe avaliadora.

Houve relatos por trabalhadores afirmando que os protocolos foram colocados nos setores no dia de nossas chegadas no hospital. (N4-42)

A instituição de protocolos de boas práticas é uma estratégia que pretende induzir mudanças nas condutas da equipe de saúde, de modo que as práticas adotadas estejam em conformidade com evidências científicas e sejam fruto de discussão coletiva de trabalhadores. Entretanto, os resultados demonstraram práticas individualizadas e dependentes de cada trabalhador de plantão.

Ao longo dos dias em que a avaliação foi realizada, foi possivel perceber que as condutas variavam de acordo com os profissionais/plantões. (N1-5)

Algumas práticas desaconselháveis são realizadas, dependendo do médico que esteja no plantão. (NE4-13)

Em relação aos indicadores, os relatos apontaram que, embora não fossem sempre utilizados, esta avaliação proporcionou a oportunidade de discussão, orientação e reposicionamento por parte dos gestores.

Os indicadores não são usados, pois não tinham a percepção de como poderiam ser usados para melhorar o serviço prestado. (N4-4)

Depois da análise documental, os gestores demonstraram o compromisso e entendimento da importância dos indicadores para o planejamento da assistência. (N4-8)

Poucos indicadores são acompanhados, mas, aproveitando o momento da avaliação e com a finalidade que tem de processo educativo, os gestores se propuseram a acompanhar os indicadores solicitados. (N4-29)

O monitoramento dos indicadores é importante para a análise dos resultados do trabalho produzido. Possibilita que as equipes corrijam rumos para uma oferta de atenção mais qualificada. A produção de uma cultura avaliativa de forma transversal aos processos de planejamento e gestão constitui-se num dos grandes desafios para os sistemas de saúde na atualidade ${ }^{34}$.

A potência da avaliação em sua dimensão indutora de transformações ficou evidente tanto nas situações em que os indicadores foram organizados e estudados para a apresentação aos avaliadores, quanto naquelas em que a equipe de avaliação ajudou os gestores a calculá-los, tornando o momento estratégico também para ações de qualificação do serviço. 
Outro elemento identificado como positivo foram os encontros produzidos no processo de avaliação. Em alguns locais, a presença de gestores das secretarias de saúde do estado e município, acompanhando o processo avaliativo induziram agendas para negociações, pactuações e compromissos.

A gestão da maternidade demonstrou desejo e compromisso com a mudança, expresso nas agendas que essa avaliação provocou entre os representantes do serviço e da gerência de saúde. Ficou pactuada, também, conversa de gestores da SES com o gestor municipal para apoiar a implementação da RC em todo o município, articulando melhor as equipes de saúde da família e da maternidade. (NE2-4)

No curso das visitas de avaliação, os representantes do serviço e da gerência de saúde pactuaram diversas agendas de apoio à qualificação do cuidado. (NE2-7)

$\mathrm{Na}$ avaliação, algumas agendas foram pactuadas para desdobramentos após o processo avaliativo. (NE2-19)

Uma das apostas metodológicas do processo avaliativo da RC foi a inclusão de diferentes atores, entre eles gestores de diferentes instâncias do SUS, não apenas para uma validação e legitimação do processo, mas, também, pela oportunidade de trocas e tomadas de decisões potencialmente transformadoras do modelo de gestão e atenção ao parto e nascimento no país.

As narrativas aqui apresentadas - produzidas a partir da escuta de gestores, trabalhadores e usuárias e a observação das práticas ao longo da avaliação de cada estabelecimento - trazem um olhar avaliativo acerca da realidade de maternidades do N e NE. A visibilidade dos contextos proporcionada pelas descrições registradas nos diários de campo e sua posterior análise possibilitou, juntamente com os resultados encontrados a partir dos questionários, a compreensão de questões prementes no que tange à saúde materna e infantil.

Uma das forças desta pesquisa qualitativa é permitir a análise da implantação da RC nas regiões de maior necessidade no país, onde persistem, há séculos, os mais graves indicadores de morbimortalidade materna e infantil. Não foram identificadas outras pesquisas avaliativas relacionadas à gestão do cuidado e atenção à saúde da mulher e da criança após a implantação da RC, envolvendo todos os estados do N e NE do Brasil.
A dificuldade de comparação dos resultados deste segundo ciclo avaliativo da RC com os do primeiro foi um dos limites deste trabalho, considerando que os procedimentos metodológicos empregados em ambos os ciclos foram muito diferentes.

\section{Considerações finais}

Foram evidenciadas mudanças em direção às boas práticas preconizadas pela Rede Cegonha, tanto na gestão quanto na atenção, mas são muitos os desafios frente ao predomínio de um modelo de gestão hierárquico associado a um modelo de atenção com práticas intervencionistas. Desta forma, apesar dos avanços produzidos com a sua implantação, ainda existe a necessidade de mais investimentos voltados para a humanização da assistência.

Considerando que esta pesquisa aconteceu no segundo ciclo de avaliações da RC, é preciso avançar para uma efetiva transformação das práticas de gestão e atenção, especialmente no que concerne à efetivação de espaços de gestão compartilhada, à capacitação de profissionais das maternidades e secretarias de saúde, à implantação de protocolos de boas práticas e à institucionalização de processos avaliativos no cotidiano do trabalho em maternidades para acompanhamento da implementação da RC. Além disso, a função do apoio institucional mostrou-se, no primeiro ciclo avaliativo, como central para a implantação das diretrizes da RC. Apostar nessa função é uma importante estratégia para a consolidação de mudanças em direção à melhoria da qualidade do cuidado.

Reforça-se a importância de que, desde o prénatal, na atenção primária, sejam realizadas ações efetivas em direção ao empoderamento das mulheres para que conheçam e se apropriem cada vez mais dos seus direitos. As equipes da atenção primária podem desenvolver um importante papel na construção do protagonismo e autonomia das mulheres a partir do investimento em processos de educação em saúde.

Os resultados desse processo avaliativo podem ser instrumentos de qualificação da gestão e da atenção nos serviços de saúde maternos e infantis. Desta forma, novos ciclos avaliativos são necessários para o monitoramento contínuo da qualificação do cuidado. 


\section{Colaboradores}

ZC Lamy, LLM Gonçalves, RH Britto, MTSSB Alves, ME Koser, MS Martins, NP Leal, EBAF Thomaz, os autores designados, assumimos publicamente a responsabilidade pelo conteúdo apresentado e declaramos que participamos igualmente de todas as etapas do artigo que incluem a análise e a interpretação dos dados, a redação das versões do artigo e a aprovação da versão final, ora apresentada.

\section{Referências}

1. Brasil. Ministério da Saúde (MS). Manual Prático para Implementação da Rede Cegonha. Brasília: Editora MS; 2011.

2. Rattner D. Da Saúde Materno Infantil ao PAISM. Tempus - Actas Saude Coletiva 2014; 8(2):103-108.

3. Cavalcanti PCS, Gurgel Junior GD, Vasconcelos ALR, Guerrero AVP. Um modelo lógico da Rede Cegonha. Physis 2013; 23(4):1297-1316.

4. Pasche DF, Vilela MEA, Giovanni MD, Almeida PVB, Franco Netto TL. Rede Cegonha: desafios de mudanças culturais nas práticas obstétricas e neonatais. $D i-$ vulgação Saude para Debate 2014; (52):58-71.

5. Pasche DF, Vilela MEA, Martins, CP. Humanização da atenção ao parto e ao nascimento no Brasil: pressupostos para uma nova ética na gestão e no cuidado. Tempus - Actas Saude Coletiva 2010; 4(4):105-117.

6. Brasil. Ministério da Saúde (MS). Departamento de Ações Estratégicas. Avaliação do apoio institucional na Rede Cegonha: avanços e desafios baseados em pesquisa avaliativa. Brasília: MS; 2016. [não publicado].

7. Leal MC, Bittencourt SA, Esteves-Pereira AP, Ayres BVS, Silva LBRAA, Thomaz EBAF, Lamy ZC, Nakamura-Pereira M, Torres JA, Gama SGN, Domingues RMSM, Vilela MEA. Avanços na assistência ao parto no Brasil: resultados preliminares de dois estudos avaliativos. Cad Saude Publica 2019; 35(7):e00223018.

8. Vilela MEA, Leal MC, Thomaz EBAF, Gomes M, Bittencourt SA, Gama SGN, Silva LBRAA, Lamy ZC Avaliação da atenção ao parto e nascimento nas maternidades da Rede Cegonha: os caminhos metodológicos. Cien Saude Colet 2021; 26(3):789-800.

9. Leal MC, Szwarcwald CL, Almeida PVB, Aquino EML Barreto ML, Barros F, Victora C. Saúde reprodutiva materna, neonatal e infantil nos 30 anos do Sistema Único de Saúde (SUS). Cien Saude Colet 2018; 23(6):1915-1928

10. Minayo MCS. O Desafio do Conhecimento: pesquisa qualitativa em saúde. $8^{\text {a }}$ ed. São Paulo, Rio de Janeiro: Hucitec, Abrasco; 2014.

11. Feuerwerker L, Cecílio L. O hospital e a formação em saúde: desafios atuais. Cien Saude Colet 2007; 12(4):965-997.

12. Deslandes S. O processo de trabalho na emergência. In: Deslandes S. Frágeis Deuses: profissionais da emergência entre os danos da violência e a recriação da vida. Rio de Janeiro: Editora Fiocruz; 2002. p. 45-94.

13. Santos-Filho SB, Barros MEB. O trabalho em saúde e o desafio da humanização: algumas estratégias de análise-intervenção. Tempus - Actas Saude 2012; 6(2):101-122.

14. Santos-Filho SB, Barros EB, Gomes RS. A Política Nacional de Humanização como política que se faz no processo de trabalho em saúde. Interface (Botucatu) 2009; 13(Supl. 1):603-613.

15. Pasche DF. Política Nacional de Humanização como aposta na produção coletiva de mudanças nos modos de gerir e cuidar. Interface (Botucatu) 2009; 13(Supl. 1):701-708.

16. Pasche DF, Passos E. A importância da humanização a partir do sistema único de saúde. Rev Saude Publ Santa Cat 2008; 1(1):92-100. 
17. Pasche DF. Pistas metodológicas para se avançar na humanização dos hospitais no Brasil. Cadernos HumanizaSUS. Brasília: Editora MS; 2011.v. 3. p. 29-50.

18. Pierantoni CR, Vianna CMM, França T, Magnago C, Rodrigues MPS. Rotatividade da força de trabalho médica no Brasil. Saude Debate 2015; 39(106):637647.

19. Zanardo GLP, Uribe MC, Nadal AHR, Habigzang LF. Violência Obstétrica no Brasil: uma revisão narrativa. Psicol Soc 2017; 29:e155043.

20. Organização Mundial da Saúde (OMS). Prevenção e eliminação de abusos, desrespeito e maus-tratos $d u$ rante o parto em instituições de saúde. Genebra: OMS; 2014. [acessado 2020 Jun 5]. Disponível em: http:// apps.who.int/iris/bitstream/10665/134588/3/WHO_ RHR_14.23_por.pdf

21. Savage V, Castro A. Measuring mistreatment of women during childbirth: a review of terminology and methodological approaches. Reprod Health 2017; 14(1):138.

22. Zaami S, Stark M, Beck R, Malvasi A, Marinelli E. Does episiotomy always equate violence in obstetrics? Routine and selective episiotomy in obstetric practice and legal questions. Eur Rev Med Pharmacol Sci 2019; 23(5):1847-1854.

23. Diniz SG, Salgado HO, Andrezzo HFA, Carvalho PGC, Carvalho PCA, Aguiar CA, Nyi DY. Violência obstétrica como questão para a saúde pública no Brasil: origens, definições, tipologia, impactos sobre a saúde materna, e propostas para sua prevenção. $J$ Hum Growth Dev 2015; 25(3):377-382.

24. Mariani A, Nascimento-Neto J. Violência obstétrica como violência de gênero e violência institucionalizada: breves considerações a partir dos direitos humanos e do respeito às mulheres. Cad Esc Direito 2016; 2(25):48-60.

25. Marrero L, Bruggemann OM. Violência institucional durante o processo parturitivo no Brasil: revisão integrativa. Rev Bras Enferm 2018; 71(3):1152-1161.

26. Robles AF. Da gravidez de "risco" às "maternidades de risco". Biopolítica e regulações sanitárias nas experiências de mulheres de camadas populares de Recife. Physis 2015; 25(1):139-169.

27. Jewkes R, Penn-Kekana L. Mistreatment of women in childbirth: time for action on this important dimension of violence against women. PLoS Med 2015; 12(6):1-4.

28. Aguiar J, D’Oliveira A, Schraiber L. Violência institucional, autoridade médica e poder nas maternidades sob a ótica dos profissionais de saúde. Cad Saude Publica 2013; 29(11):2287-2296.
29. Anunciação PSD, Lamy ZC, Pereira MUL, Madeira HGR, Loyola CD, Gonçalves LLM, Lamy-Filho F. "A tragedy after giving birth": stories of women who have lost newborn children. Cad Saude Publica 2018; 34(12):e00190517.

30. Diniz CSG, Rattner D, D'Oliveira AFPL, Aguiar JM, Niy DY. Disrespect and abuse in childbirth in Brazil: social activism, public policies and providers' training. Reprod Health Matters 2018; 26(53):19-35.

31. Diniz CSG, Niy D, Andrezzo H, Carvalho P, Salgado H. A Vagina-escola: seminário interdisciplinar sobre violência contra a mulher no ensino das profissões de saúde. Interface - (Botucatu) 2016; 20(56):253-259.

32. Brasil. Ministério da Saúde (MS). Portaria No 1.153, de 22 de maio de 2014. Redefine os critérios de habilitação da Iniciativa Hospital Amigo da Criança (IHAC), como estratégia de promoção, proteção e apoio ao aleitamento materno e à saúde integral da criança e da mulher, no âmbito do Sistema Único de Saúde (SUS). Diário Oficial da União 2014; 22 maio.

33. Carvalho ALB, Souza MF, Shimizu HE, Senra IMVB, Oliveira KC. A gestão do SUS e as práticas de monitoramento e avaliação: possibilidades e desafios para a construção de uma agenda estratégica. Cien Saude Coletiva 2012; 17(4):901-911.

34. Jardim DMB, Modena CM. Obstetric violence in the daily routine of care and its characteristics. Rev Lat Am Enferm 2018; 26:e3069.

Artigo apresentado em 23/04/2020

Aprovado em 31/07/2020

Versão final apresentada em 02/08/2020

Editores-chefes: Romeu Gomes, Antônio Augusto Moura da Silva 